\title{
Guidance for footbridge design: a new simplified method for the accurate evaluation of the structural response in serviceability conditions
}

\author{
Caterina Ramos-Moreno ${ }^{1}$ (D), Ana M. Ruiz-Teran ${ }^{2 *}$ and Peter J. Stafford ${ }^{2}$
}

\author{
${ }^{*}$ Correspondence: \\ a.ruiz-teran@imperial.ac.uk \\ ${ }^{2}$ Department of Civil and \\ Environmental Engineering, \\ Imperial College London, South \\ Kensington Campus, SW7 2AZ \\ London, United Kingdom \\ Full list of author information is \\ available at the end of the article
}

\begin{abstract}
This paper proposes a simplified hand-calculation methodology that permits a fast response assessment (both in vertical and lateral direction) under different pedestrian scenarios. This simplified method has the same accuracy than that of very sophisticated numerical nonlinear finite element models including pedestrian inter-variability, interaction among pedestrians in flows, and pedestrian-structure interaction. The method can capture the effects of pedestrian loads in and out of resonance. This methodology is based on a new, and experimentally contrasted, stochastic pedestrian load model derived by the authors implementing a multi-disciplinary state-of-the-art research, and on a large set of sophisticated finite element analyses.

There is a significant gap in the literature available for bridge designers. Some current codes do not indicate how the performance for serviceability limit-states should be assessed, in particular for lateral direction. Others define methods that are not based on the latest research in this field and that require the use of dynamic structural analysis software. A very sophisticated load model, such as that described above, and recently proposed by the authors, may not be accessible for most of the design offices, due to time and software constraints. However, an accurate assessment of the serviceability limit state of vibrations during the design stages is paramount. This paper aims to provide designers with an additional simple tool for both preliminary and detailed design for the most typical structural configurations.

First, the paper presents the methodology, followed by an evaluation of the impact of its simplifications on the response appraisal. Second, the paper evaluates the validity of the methodology by comparing responses predicted by the method to those experimentally measured at real footbridges. Finally, the paper includes a parametric analysis defining the maximum accelerations expected from pedestrian streams crossing multiple footbridges. This parametric analysis considers different variables (Continued on next page)
\end{abstract}

(C) The Author(s). 2020 Open Access This article is licensed under a Creative Commons Attribution 4.0 International License, which permits use, sharing, adaptation, distribution and reproduction in any medium or format, as long as you give appropriate credit to the original author(s) and the source, provide a link to the Creative Commons licence, and indicate if changes were made. The images or other third party material in this article are included in the article's Creative Commons licence, unless indicated otherwise in a credit line to the material. If material is not included in the article's Creative Commons licence and your intended use is not permitted by statutory regulation or exceeds the permitted use, you will need to obtain permission directly from the copyright holder. To view a copy of this licence, visit http://creativecommons.org/licenses/by/4.0/. 
(Continued from previous page)

such as section type, structural material, span length and traffic-flow characteristics, and shows the sensitivity of the serviceability response to traffic-flow characteristics and span length in particular.

Keywords: Footbridge, Pedestrian load, Vibration, Serviceability, Vertical response, Lateral response

\section{Introduction}

Footbridge design has evolved rapidly in recent decades, leading designers to use new and lighter materials (Firth and Cooper 2002) and structural arrangements that allow for the use of less massive cross sections and longer spans. However, it is well-known that this evolution in design has produced bridges where serviceability requirements were not satisfied (e.g., the London Millennium Bridge, Dallard et al. 2001, or the Solférino Bridge, currently called Léopold-Sédar-Senghor, in Paris). Nonetheless, this progression in design is not the sole cause of these serviceability issues. Detailed studies of these events have demonstrated that assumptions made to represent serviceability events with pedestrian flows were not sufficiently realistic.

Designers need to be able to predict, from the very early stages of their designs, whether or not their proposals can satisfy the serviceability requirements (by comparing maximum predicted accelerations of the structure in service to limits of acceptance, established according to human perception of movement). Most structural design codes, e.g., EN 1991-2:2003 (2003) \& AASHTO (2009), advise that serviceability limit-states may become critical if structural frequencies are within the ranges of common pedestrian step frequencies (around 1.0-3.0 Hz for vertical vibrations, and 0.5-1.5 Hz for lateral vibrations). However, many of these codes do not define the procedure that should be adopted to perform these analyses, e.g., AASHTO (2009) \& CAN/CSA-S6-06 (2006). For instance, the dynamic load models required for verifications according to Eurocode 1 (2003) are not defined in the main body of the document, and are instead left to be defined in each national annex. In some cases, additional specifications have been defined (e.g., NA to BS EN 1991-2:2003, 2008, for the UK), but this is not always the case. In addition, these code methods for serviceability evaluation have two main disadvantages: on one hand, they are based on load models that do not include the latest research advances related to this field and, on the other hand, they are computationally demanding (not being useful for preliminary design). Related to the first point, many codes or design guidelines still apply several assumptions similar to those proposed in early codes, such as BS 5400 (1978). This is undoubtedly due to two facts: (1) the current state-of-the-art of all the aspects related to pedestrian loading is still very dispersed within the literature; and (2) a modern methodology including the latest research advances and using the latest cutting-edge analyses to accurately represent reality has not been published yet.

With the aim of providing a tool to perform this serviceability assessment, and based on the latest research, this paper proposes a very simple method to accurately obtain the maximum vertical and lateral accelerations expected in a footbridge due to pedestrian actions. The method allows the designer to verify the serviceability limit state of vibrations due to traffic load without performing the corresponding, sophisticated, timeconsuming, and computationally-demanding analyses. The simple method presented 
here is underpinned by a comprehensive, accurate, and realistic description of pedestrian loads defined by the authors, that are well beyond those conventionally adopted in current practise (including intra-variability, inter-variability, pedestrian-pedestrian interactions and pedestrian-structure interactions among others). This pedestrian load model defined by the authors is based on existing, but very dispersed research works that have been published over recent years. The method presented in this paper is therefore very useful both for detailed and preliminary design.

The method is applicable to footbridges of one-to-three spans, with constant depth. However, it can easily be adopted for footbridges with a larger number of spans, nonconstant depth, or more sophisticated geometry. The response is obtained based on the structural features of the footbridge (such as the geometry of the section, and material properties) and traffic-flow characteristics (such as the travel purpose, and pedestrian density). An assessment of the performance of the method is included.

The soundness of the proposed method is appraised by comparing the serviceability response produced by the method for several real footbridges to those experimentally recorded at the same footbridges. Finally, based on the method introduced, the paper evaluates the performance in serviceability in the vertical or lateral directions of a wide range of footbridges with one or two spans. These evaluations are defined for structures designed with different material and geometrical configurations.

\section{Conceptual basis for the proposed method}

The overall method presented over the course of the following sections is based upon a very simple conceptual model. The general idea is that the response of footbridges under serviceability conditions is essentially a reflection of the resonant characteristics that arise from the interaction of the structural properties and the pedestrian loading characteristics. A physically-based equation is therefore developed that identifies the key frequency ranges that will be most important for the acceleration response of simple bridges in service conditions. This equation is parameterised using very common and fundamental geometric and material properties and can therefore be applied to a very wide range of structural configurations.

This governing equation is considered in the actual appraisal of a bridge response under pedestrian actions taking into consideration the Pi Theorem of Dimensional Analysis (Buckingham 1914). This theorem ensures that a function that describes a physical problem $f(\boldsymbol{x})=f\left(x_{1}, x_{2}, \ldots, x_{n}\right)=0$, with $n$ arguments that are defined with respect to $q$ fundamental units $U_{1}, U_{2}, \ldots, U_{q}$, can be represented as $g\left(\pi_{1}, \pi_{2}, \ldots, \pi_{k}\right)=0$, where $k<n$. In this second function $k=n-r$, and $r$ is the rank of the dimensional matrix $n \times q$. The $\pi$ terms are independent and dimensionless products formed from the original $n$ variables $x_{1}, \ldots, x_{n}$.

For our particular case, $f(\boldsymbol{x})=0$ is the function that predicts the structural response, which arises from the equation of motion of a dynamic system. For the case of vertical accelerations in a simply-supported bridge, this is a function of both the structural properties (the structural mass, the span length, the material damping ratio, and the flexural stiffness) and the pedestrian characteristics (the pedestrian mass, their step frequency, and their step length) and provides a prediction of the expected maximum acceleration of the bridge. Therefore, there are seven input variables used to predict one response variable (eight variables in total in implicit function $f(\boldsymbol{x})$, i.e., $n=8$ ). However, when the 
lateral accelerations are considered two additional parameters are required and these are the pedestrian step width and the height of the pedestrian, meaning that in the lateral case $n=10$.

This physical problem involves the three fundamental units of mass, length and time (which we define as $U_{1}, U_{2}$ and $U_{3}$ ). To identify the required number of nondimensional parameters it is necessary to represent each of our 8 (or 10) variables in terms of these three basic units through an $n \times q$ matrix. The difference between the total number of variables and the rank of this matrix then defines the required number of nondimensional parameters. In the present case, with the fundamental units defined as above, the rank of this matrix will be $r=3$ and will imply that five (or seven) $\pi$ terms are required. Four (or six) of these terms relate to the input variables while one term is associated with the response, and is the normalised acceleration $a / g$, with $g$ being the gravitational acceleration of the Earth - considered to be a constant.

The dimensionless parameters are then chosen to be the ratio of the frequency of the structure to the pedestrian step frequency, $\pi_{1}=f_{s} / f_{p}$, the ratio of the structural mass to the pedestrian mass, $\pi_{2}=m_{s} / m_{p}$, the ratio of the pedestrian step length and the span length $\pi_{3}=s l / L$, and the damping ratio, $\pi_{4}=\zeta$. For the lateral case $\pi_{5}$ and $\pi_{6}$ are also used and are set as pedestrian step widths and heights normalized by corresponding values representative of the target population.

Considering a range of values for the first dimensionless term $\left(f_{s} / f_{p}\right)$ and specific representative values for the remaining dimensionless terms, we then generate a very large number of results from advanced numerical analyses using sophisticated finite-element models, with realistic descriptions of the pedestrian loading that account for the most pertinent features of the loading in both vertical and lateral directions. These numerical results are then employed to determine basic or reference vertical and lateral accelerations (listed in the Additional files 1 and 2 of the paper), that correspond to the particular structural and pedestrian characteristics used for the sophisticated numerical analyses. Given the scope of the present study, the basic accelerations are defined directly in units of $\mathrm{m} / \mathrm{s}^{2}$, computed from $a / g$ using $g=9.81 \mathrm{~m} / \mathrm{s}^{2}$.

Naturally, however, we need to ensure that the method works for a broad range of configurations and not just the reference cases obtained for particular values of $\pi_{2}, \pi_{3}, \pi_{4}$, $\pi_{5}$ and $\pi_{6}$. We therefore derive a series of adjustment factors that act to scale these basic accelerations in order to account for departures from the original values of $\pi_{2, \ldots, 6}$.

In the sections that follow the approach to determine these basic accelerations is first defined before explaining and presenting the various adjustment factors that can be applied to adapt these basic values to be appropriate for another structural and loading configuration of interest.

\section{Realistic description of the pedestrian loading}

When walking, individuals introduce vertical and horizontal forces on the surface upon which they are supported (see Fig. 1a). The definition of the forces exerted is complex and involves many parameters. However, pedestrian loading is currently defined in many codes and guidelines in quite a simplistic manner. The main simplifications in the load description are related to the step frequency and the load amplitude.

Current methods are based on descriptions where all the individuals walk with frequencies with values of approximately 1.8 to $2.0 \mathrm{~Hz}$ (see e.g., Pedersen and Frier, 2010). 


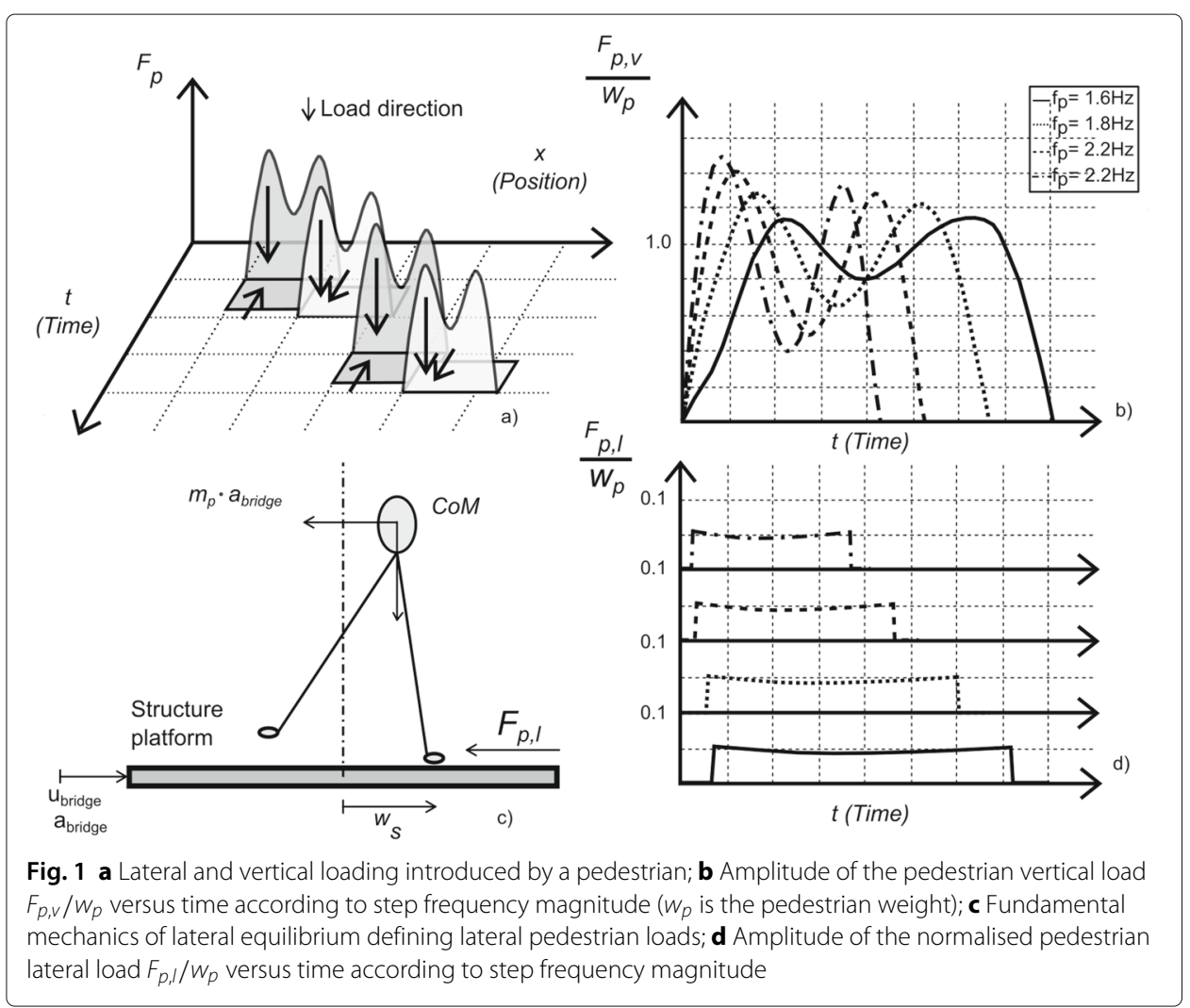

However, research has shown that the distribution of pedestrian step frequencies of a crowd is not fixed and depends on many parameters. Bertram and Ruina (2001) showed a clear correlation between step frequency and pedestrian speed, based on experimental tests, while Weidmann (1993) showed that pedestrian speed is chosen according to aspects such age, anthropometric characteristics, aim of the journey, and pedestrian density, among others. Accordingly, an accurate description of the pedestrian load needs to take these issues into consideration. Therefore, the authors have proposed probabilistic relationships (Ramos Moreno et al. 2020) to define pedestrian target speed and frequency. The target speed of a pedestrian is defined based on characteristics, such as age and height, the aim of journey (considering three different categories business: that includes journeys related to work for pedestrians with an age range between 20 and 65 years old, but excludes their commute to and from home; commuter: which defines journeys between work/study place and home of pedestrians, regardless their age; and, leisure: that includes shopping, tourist or leisure-related journeys), and the density of the crowd in which they walk (number of pedestrians per unit area). The pedestrian frequency is obtained based on the pedestrian speed. By means of these fundamental relationships, based on the UK and Western Europe population data, the distribution of step frequencies according to aim and density of the flow have been defined (Fig. 2).

In codes and guidelines, load amplitudes are usually described by means of Fourier series with a single term (a sinusoidal function) representing the load transmitted by both feet in time. In these methods, the maximum load amplitude is a function of the pedestrian weight and is independent of the gait, which is not realistic. In addition, several of these methods use the same mathematical definition for both lateral and vertical loads, 


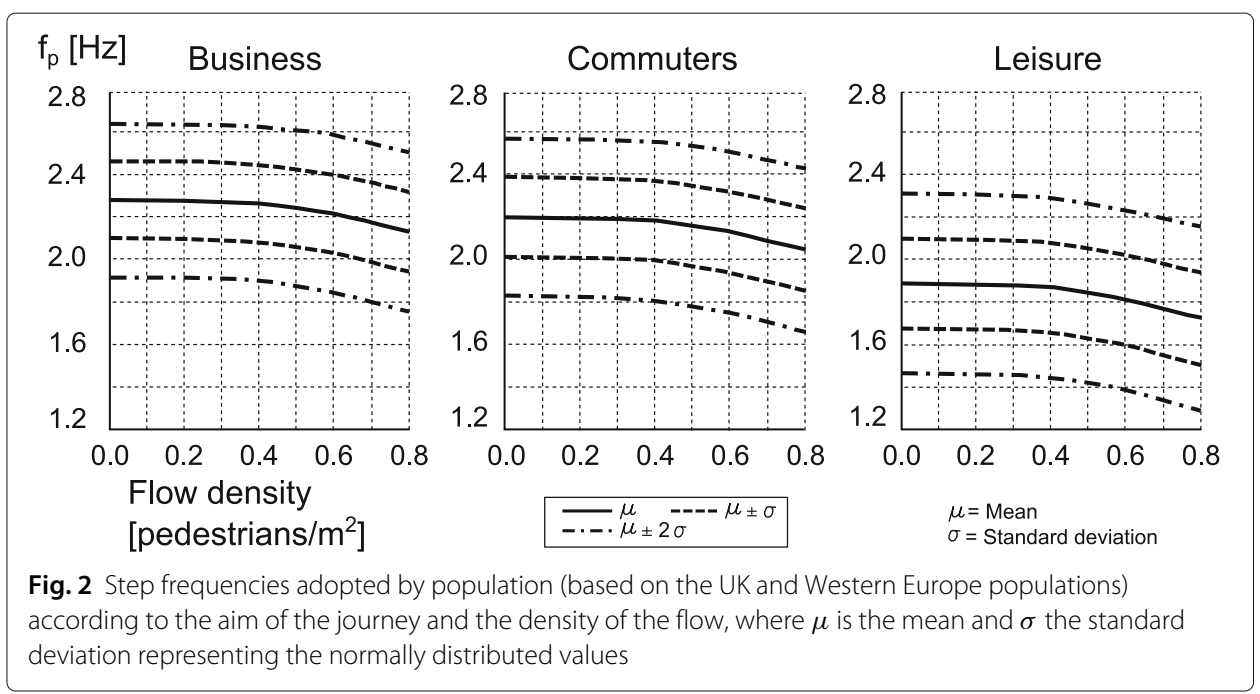

which does not reproduce the nonlinear pedestrian-structure interaction that has been detected in real structures for lateral movement (e.g., Dallard et al. 2001).

The work presented in this paper has been obtained using a realistic and experimentally contrasted description of the pedestrian loading, defining each foot load individually as illustrated in Fig. 1a. The model describing vertical and lateral loads individually transmitted by each foot of a pedestrian has been derived by the authors of this paper and an extensive description can be found in Ramos Moreno et al. (2020). The model considers pedestrian inter-variability, interaction among pedestrians in flows and pedestrian-structure interaction among others. The functions used to define the vertical load amplitude (Fig. 1b) are based on the work published by Butz et al. (2008). Their definition is exclusively based on the step frequency (which has been linked herein to those parameters considered in Fig. 2). The lateral loads are based on a model that reproduces the movement of the centre of mass of each pedestrian (Macdonald 2009). This approach has been adopted primarily due to its foundation in fundamental mechanics (Fig. 1d). This model for lateral loads has been shown to compare relatively well with empirical data, although studies of lateral loads are characterised by a great deal of variability and inconsistency. This definition is also convenient for considering the nonlinearity attributed to these lateral loads, since they seem to be related to the lateral movement perceived by the individual (Dallard et al. 2001). The longitudinal loading introduced by the pedestrian has been neglected, as longitudinal vibrations are typically not important when assessing performance in serviceability.

\section{Vertical and lateral structural frequencies}

For a simply-supported beam of constant geometrical and mechanical characteristics throughout the length, the vertical, $f_{v, n}$, and lateral, $f_{l, n}$, vibration frequencies associated with the $n^{\text {th }}$ vertical and lateral vibrational modes are given by Eq. 1 :

$$
f_{y, n}=\frac{n^{2} \pi}{2 L^{2}} \sqrt{\frac{E I_{y}}{\rho A}}, \quad y \in\{v, l\}
$$


where $L$ is the span length, $E$ is Young's modulus, $\rho$ is the material density, $A$ is the crosssectional area of the section, and $I_{x}, x \in\{v, l\}$, are the second moments of area in vertical and lateral direction of the section, respectively.

The ratio between the second moment of area, in the vertical and lateral directions, and the cross sectional area, can be defined by Eqs. 2 and 3:

$$
\begin{aligned}
& \frac{I_{v}}{A}=\eta_{\nu} \alpha_{\nu}\left(1-\alpha_{v}\right) h^{2} \\
& \frac{I_{l}}{A}=\eta_{l} \alpha_{l}\left(1-\alpha_{l}\right) b^{2}
\end{aligned}
$$

where $\alpha_{v}$ is the ratio between the vertical distance from the centroid of the section to the top extreme fibre and the vertical depth of the section $h ; \alpha_{l}$ is the ratio between the horizontal distance from the centroid of the section to the closest lateral extreme fibre and the width of the section $b ; \eta_{v}$ is the ratio between the depth of the central kern and the depth of the section $h$; and $\eta_{l}$ is the ratio between the width of the central kern and the width of the structural section $b$.

By substituting Eqs. 2 and 3 into Eq. 1, the following expressions are obtained:

$$
\begin{aligned}
& f_{v, n}=\frac{n^{2} \pi}{2 L} \sqrt{\frac{E}{\rho} \eta_{\nu} \alpha_{v}\left(1-\alpha_{v}\right)\left(\frac{h}{L}\right)^{2}} \\
& f_{l, n}=\frac{n^{2} \pi}{2 L^{2}} \sqrt{\frac{E}{\rho} \eta_{l} \alpha_{l}\left(1-\alpha_{l}\right) b^{2}}
\end{aligned}
$$

The parameters $\eta_{v}, \eta_{l}, \alpha_{v}$ and $\alpha_{l}$ take reasonably constant values for each section type. Figure 3 provides values of these parameters for conventional sections that can be used in footbridge design (values derived from existing footbridges and from values typically used by bridge engineers).

In preliminary design, the vertical and lateral frequencies of the structure can be directly estimated from Eqs. 4 and 5, whilst in detailed design they can be estimated from Eq. 1 or directly obtained from finite element (FE) models. For lateral frequencies, Eq. 1 describes the vibration modes of bridges where bearings allow the rotation with respect to the line described by the lateral centre of gravity of the section (for other bearing dispositions FE models will provide a more accurate evaluation of the modal vibration).

\section{Resonance parameters}

The main parameters that control the vertical and lateral response of a footbridge under pedestrian loading are the ratios between the vertical or lateral structural frequencies and the corresponding pedestrian frequencies. The ratio $r_{v, n}$ between the $n^{\text {th }}$ vertical structural frequency and the pedestrian vertical frequency $f_{p, v}$ (or simply $f_{p}$, hereafter), is given by Eq. 6, in which $\phi_{s, n}$ is an adjustment factor to account for cases that differ from a simply-supported bridge, and $\rho^{*}$ differs from $\rho$ as it also considers the additional nonstructural mass. Note that the resonance parameters being discussed here are equivalent to the first nondimensional parameter $\pi_{1}$ presented earlier in the case that $\phi_{s, n}=1$ and $\rho^{*}=\rho$.

$$
r_{v, n}=\frac{f_{v, n}}{f_{p, v}} \phi_{s, n}=\frac{n^{2} \pi}{2 L f_{p}} \sqrt{\frac{E}{\rho^{*}} \eta_{\nu} \alpha_{\nu}\left(1-\alpha_{v}\right)\left(\frac{h}{L}\right)^{2}} \phi_{s, n}
$$




\begin{tabular}{|c|c|c|c|c|}
\hline & \multicolumn{2}{|c|}{ Geometric efficiency } & \multirow{2}{*}{$\begin{array}{c}\text { Usual } \\
\text { and new } \\
\text { Material }\end{array}$} & \multirow{2}{*}{$\begin{array}{c}\text { Common } \\
\text { Generic dimensions }\end{array}$} \\
\hline & Vertical & Lateral & & \\
\hline$\stackrel{\mathrm{b}}{\square}$ Ih & $\begin{array}{l}\eta_{v}=0.333 \\
\alpha_{v}=0.500\end{array}$ & $\begin{array}{l}\eta_{\mathrm{h}}=0.333 \\
\alpha_{\mathrm{h}}=0.500\end{array}$ & $\begin{array}{l}\text { Reinforced } \\
\text { Concrete } \\
\text { Prestressed } \\
\text { Concrete } \\
\end{array}$ & $\begin{array}{c}1 / 25<\mathrm{h} / \mathrm{L}<1 / 20 \\
10<\mathrm{L}<20 \mathrm{~m} \\
1 / 30<\mathrm{h} / \mathrm{L}<1 / 25 \\
15<\mathrm{L}<30 \mathrm{~m}\end{array}$ \\
\hline$\sqrt{ } \mathrm{h}$ & $\begin{array}{l}\eta_{v}=0.360 \\
\alpha_{v}=0.430\end{array}$ & $\begin{array}{l}\eta_{h}=0.240 \\
\alpha_{h}=0.500\end{array}$ & Concrete & $\begin{array}{l}1 / 30<\mathrm{h} / \mathrm{L}<1 / 25 \\
15<\mathrm{L}<30 \mathrm{~m}\end{array}$ \\
\hline$\perp^{h}$ & $\begin{array}{l}\eta_{v}=0.570 \\
\alpha_{v}=0.390\end{array}$ & $\begin{array}{l}\eta_{\mathrm{h}}=0.240 \\
\alpha_{\mathrm{h}}=0.500\end{array}$ & Concrete & $\begin{array}{l}1 / 35<h / L<1 / 30 \\
35<L<55 m\end{array}$ \\
\hline - & $\begin{array}{l}\eta_{v}=0.525 \\
\alpha_{v}=0.340\end{array}$ & $\begin{array}{l}\eta_{h}=0.215 \\
\alpha_{h}=0.500\end{array}$ & $\begin{array}{l}\text { Steel - } \\
\text { Concrete }\end{array}$ & $\begin{array}{l}1 / 35<\mathrm{h} / \mathrm{L}<1 / 30 \\
30<\mathrm{L}<55 \mathrm{~m}\end{array}$ \\
\hline$\perp \perp \perp^{h}$ & $\begin{array}{l}\eta_{v}=0.405 \\
\alpha_{v}=0.340\end{array}$ & $\begin{array}{l}\eta_{\mathrm{h}}=0.205 \\
\alpha_{\mathrm{h}}=0.500\end{array}$ & $\begin{array}{l}\text { Steel - } \\
\text { Concrete }\end{array}$ & $\begin{array}{l}1 / 25<\mathrm{h} / \mathrm{L}<1 / 20 \\
25<\mathrm{L}<50 \mathrm{~m}\end{array}$ \\
\hline$b=2 b^{\prime}$ & $\begin{array}{l}\eta_{v}=0.333 \\
\alpha_{v}=0.500\end{array}$ & $\begin{array}{l}\eta_{\mathrm{h}}=0.815 \\
\alpha_{\mathrm{h}}=0.500\end{array}$ & Concrete & $\begin{array}{l}1 / 25<\mathrm{h} / \mathrm{L}<1 / 20 \\
10<\mathrm{L}<25 \mathrm{~m}\end{array}$ \\
\hline $\mathrm{b}$ & $\begin{array}{l}\eta_{v}=0.625 \\
\alpha_{v}=0.500\end{array}$ & $\begin{array}{l}\eta_{\mathrm{h}}=0.715 \\
\alpha_{\mathrm{h}}=0.500\end{array}$ & Aluminium & $\begin{array}{c}1 / 30<\mathrm{h} / \mathrm{L}<1 / 25 \\
10<\mathrm{L}<20 \mathrm{~m}\end{array}$ \\
\hline ப & $\begin{array}{l}\eta_{v}=0.333 \\
\alpha_{v}=0.500\end{array}$ & $\begin{array}{l}\eta_{\mathrm{h}}=0.460 \\
\alpha_{\mathrm{h}}=0.500\end{array}$ & Timber & $\begin{array}{l}\mathrm{h} / \mathrm{L}<1 / 20 \\
7<\mathrm{L}<20 \mathrm{~m}\end{array}$ \\
\hline $\begin{array}{l}\underset{b}{ } I_{b} I \\
\text { (*): decks whose mass } \\
\text { is not structural }\end{array}$ & $\begin{array}{l}\eta_{v}=0.700 \\
\alpha_{v}=0.500\end{array}$ & $\begin{array}{l}\eta_{\mathrm{h}}=0.450 \\
\alpha_{\mathrm{h}}=0.500\end{array}$ & $\begin{array}{l}\text { Glass Fibre } \\
\text { Reinforced } \\
\text { Polymers }\end{array}$ & $\begin{array}{l}\mathrm{h} / \mathrm{L}<1 / 25 \\
7<\mathrm{L}<20 \mathrm{~m}\end{array}$ \\
\hline
\end{tabular}

Fig. 3 Summary of geometric properties, and usual materials and span ranges for different footbridge sections. The slab defining the decking contributes to the longitudinal stiffness in sections S.1 to S.5, whereas in sections S.6-S.9 it only contributes to the transverse stiffness

When walking, consecutive vertical pedestrian loads have the same sign (downwards) and are characterised by a frequency $f_{p, v}=f_{p}$ (step frequency). For lateral loads, consecutive steps have opposite signs, corresponding to loads whose frequency is half the step frequency $\left(f_{p, l}=f_{p} / 2\right)$. The ratio between the $n^{\text {th }}$ lateral structural frequency and the lateral pedestrian frequency $f_{p, l}$, is therefore denoted by $r_{l, n}$ as in Eq. 7 .

$$
r_{l, n}=\frac{f_{l, n}}{f_{p, l}} \phi_{s, n}=\frac{n^{2} \pi}{L^{2} f_{p}} \sqrt{\frac{E}{\rho^{*}} \eta_{l} \alpha_{l}\left(1-\alpha_{l}\right) b^{2}} \phi_{s, n}
$$

When the parameters $r_{v, n}$ or $r_{l, n}$ are equal to 1 , it means that the vertical or lateral pedestrian loading is inducing resonance in the structure, as the pedestrian frequency is equal to the $n^{\text {th }}$-mode structural frequency. When these parameters are equal to 2, it means that the pedestrian loading reinforces the displacements in the $n^{\text {th }}$ mode in every other cycle. In general, when the parameters $r_{v, n}$ or $r_{l, n}$ are equal to a natural number $p$, 
the pedestrian loading will reinforce any existing structural movement at every $p$ cycles of the $n^{\text {th }}$-mode.

In the above expressions, $\rho^{*}$ is the effective material density when the non-structural mass per unit length $m$ (accounting for the pavement, parapets, and handrail weight, as well as the mass of a pedestrian stream) is also considered:

$$
\rho^{*}=\rho \cdot\left(1+\frac{m}{\rho \cdot A}\right)
$$

The parameter $\phi_{s, n}$ is related to the number of spans and the geometrical arrangement. For simply-supported spans $\phi_{s, n}=1$. For two and three span beams, this factor can be directly obtained from Fig. 4.

The pedestrian frequency $f_{p}$ should be defined based on the density of the crowd flow and the type of use expected (Fig. 2), considering that pedestrians in a flow have an effect on others. For simple calculations, the mean values of frequencies should be considered (given by the continuous line in Fig. 2). For more detailed calculations, a wider range of pedestrian frequencies should be considered (for this purpose, additional fractiles of the frequency distribution are shown in Fig. 2).

\section{Basic vertical and lateral accelerations}

The basic accelerations $\left(a_{y, n}^{b} \quad y \in\{v, l\}\right)$ linked to the $n^{\text {th }}$ mode of vibration are those associated with the passage of a single pedestrian crossing a simply-supported bridge. As mentioned in previous sections, these basic accelerations have been obtained for broad ranges of the resonance parameters and fixed values of the remaining dimensionless parameters (values are indicated in following sections).

The values of the basic accelerations (as well as accelerations obtained with any other value of the nondimensional parameters) have been obtained representing the beam structures as finite-element models with one-dimensional elements where the nodal response caused by the dynamic load created by the pedestrians crossing the structure has been appraised implementing a step-by-step time-history analysis that accounts for pedestrian-structure interaction. Results obtained with the developed numerical model have been validated with Abaqus (2013).

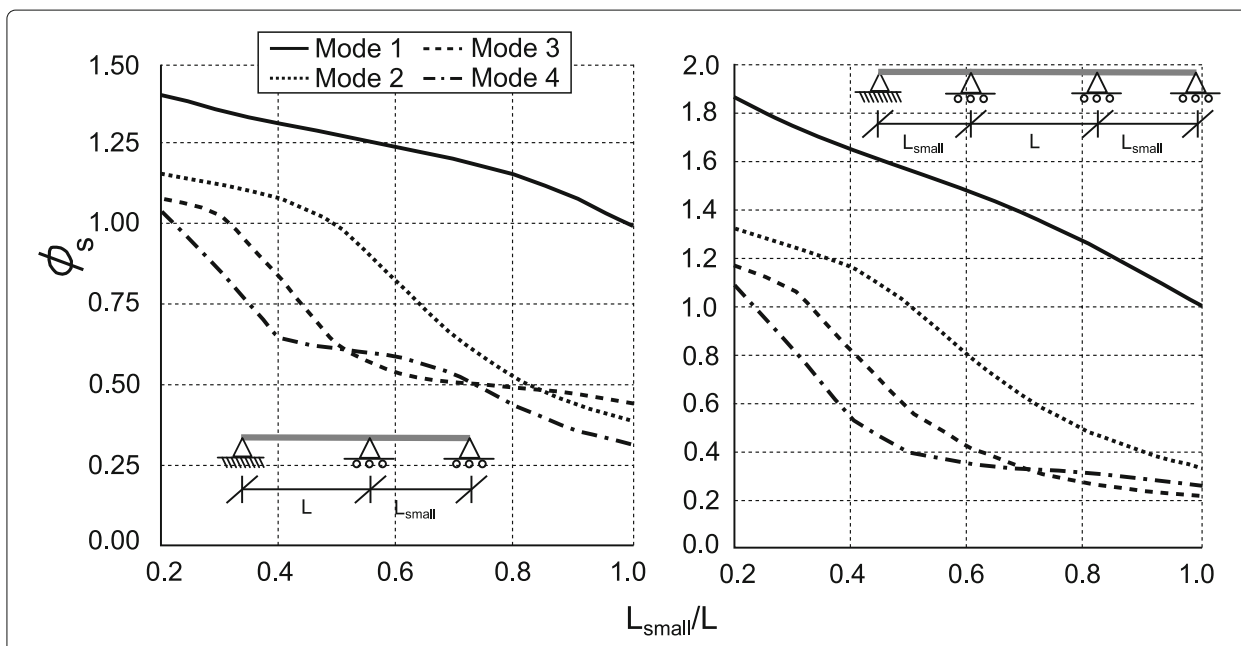

Fig. 4 Amplitude of $\phi_{s, n}$, according to mode, $n$, and number of spans 
The basic vertical acceleration linked to the $n^{\text {th }}$ mode of vibration $\left(a_{v, n}^{b}\right)$ is obtained as a function of the vertical resonance parameter $\left(r_{v, n}\right)$ and the pedestrian frequency $f_{p}$. The different load shape, and therefore impulse, for different step frequencies explains the different accelerations according to $f_{p}$ (the vertical load model derived by the authors to develop this method describes load shapes that change according to the pedestrian step frequency, see Ramos et al., 2020). The basic lateral acceleration linked to the $n^{\text {th }}$ mode of vibration $\left(a_{l, n}^{b}\right)$ is obtained as a function of the lateral resonance parameter $\left(r_{l, n}\right)$, but is independent of the step frequency (lateral loads have the same shape and impulse regardless $f_{p}$, given particular values of step width and pedestrian height, see Ramos et al., 2020). The values of these functions are listed in the Additional files 1 and 2 of the paper. Intermediate values not listed in this table can be obtained by linear interpolation. Basic vertical and lateral accelerations for vertical and lateral resonance parameters not included in the table can be assumed equal to zero.

\section{Maximum vertical and lateral accelerations caused by a single pedestrian}

The maximum vertical $\left(a_{v}\right)$ and lateral $\left(a_{l}\right)$ accelerations caused by one pedestrian are given by the expressions in Eqs. 9 and 10. These maximum accelerations are the maximum accelerations calculated from the consideration of the first four modes included in the analyses, i.e., $n=1,2,3,4$. This structural response appraisal has been considered a reliable evaluation of the total response since results show that in each case the response is largely dominated by a single vibration mode. Therefore, the results obtained using this simple approach would not differ much from more elaborate modal combination rules.

$$
\begin{aligned}
& a_{v}=\max _{n}\left(a_{v, n}^{b} \phi_{p m} \phi_{s l} \phi_{d} \phi_{s m}\right) \\
& a_{l}=\max _{n}\left(a_{l, n}^{b} \phi_{p m} \phi_{s w} \phi_{p h} \phi_{s l} \phi_{d} \phi_{s m}\right)
\end{aligned}
$$

where $\phi_{p m}$ and $\phi_{p h}$ are factors related to the mass and height of the pedestrian; $\phi_{s l}$ and $\phi_{s w}$ are factors related the length and width of the pedestrian step; $\phi_{d}$ is a factor related to the damping of the structure; and $\phi_{s m}$ is a factor related to the mass of the structure.

\subsection{Factor related to the pedestrian mass $\left(\phi_{p m}\right)$}

Codes and guidelines usually consider a standard pedestrian weight of $700 \mathrm{~N}$ (a standard pedestrian mass $m_{p}$ of $71.36 \mathrm{~kg}$ ). The basic accelerations have been obtained for a footbridge-to-pedestrian mass ratio of 7440 . For ratios different to this particular value, the maximum acceleration can be obtained from the basic acceleration by using the factor $\phi_{p m}$, which is defined by Eq. 11 .

$$
\phi_{p m}=7440 \frac{m_{p}}{\rho^{*} A L}
$$

\subsection{Factor related to the pedestrian step length $\left(\phi_{s l}\right)$}

The pedestrian step length depends on various parameters, such as speed, gender, height, etc. Values reported by Pachi and Ji (2005) suggest that a value of $0.70 \mathrm{~m}$ can be considered as representative. The basic accelerations have been obtained for a ratio between the pedestrian step and the span length of 0.02. For different ratios, the maximum acceleration can be obtained from the basic acceleration by using the factor $\phi_{s l}$, which is defined by Eq. 12 and the coefficients presented in Tables 1 and 2. 
Table 1 Coefficients for obtaining $\phi_{s /}$ in Eq. 12 for vertical response $(y=v)$, where $x$ is the ratio between the pedestrian step and the span length, $i$ is a natural number greater than 2

\begin{tabular}{lll}
\hline $\boldsymbol{r}_{\boldsymbol{v}, \boldsymbol{n}}$ interval & $\boldsymbol{B}_{\mathbf{1}}$ & $\boldsymbol{B}_{\mathbf{2}}$ \\
\hline$[0.975,1]$ & $5460 x^{2}-820 x+14.34$ & 0.975 \\
{$[1,1.025]$} & & 1.025 \\
{$[1.975,2]$} & $1180 x^{2}-215 x+3.84$ & 1.975 \\
{$[2,2.025]$} & & 2.025 \\
{$[i-0.025, i]$} & $101 x^{2}-58.5 x+1.12$ & $i-0.025$ \\
{$[i, i+0.025]$} & & $i+0.025$ \\
\hline
\end{tabular}

$$
\phi_{s l}=\exp \left(B_{1} \times\left|r_{y, n}^{2}-B_{2}^{2}\right|\right), \quad y \in\{v, l\}
$$

In deriving these factors, our results have indicated that the maximum accelerations registered are sensitive to the value of this parameter only near resonance (i.e., when the resonance parameters take values very close to a natural number) and only for the first and second vibrational modes. Therefore, this factor $\phi_{s l}$ needs to be assessed when the vertical or lateral resonance parameters linked to the first $\left(r_{v, 1}, r_{l, 1}\right)$ or the second mode $\left(r_{v, 2}, r_{l, 2}\right)$ are in the following intervals: $[i \pm 0.025]$, where $i$ is a natural number (i.e., $i=1,2,3, \ldots$ ). For all other cases, $\phi_{s l}=1$.

\subsection{Factor related to the pedestrian step width $\left(\phi_{s w}\right)$}

The pedestrian step width varies significantly among different pedestrians as well as for a given pedestrian while they walk. Despite this, a value of $w_{s}=0.10 \mathrm{~m}$ is taken as being representative for the UK and Western Europe populations (Ramos Moreno et al. 2020). This value represents the total transverse distance between feet, in units of metres. For different pedestrian step widths, the maximum acceleration can be obtained from the basic acceleration by using the factor $\phi_{s w}$, which is defined by Eq. 13 .

$$
\phi_{s w}=\frac{w_{s}}{0.10}
$$

\subsubsection{Factor related to the pedestrian height $\left(\phi_{p h}\right)$}

The basic accelerations have been obtained using a distribution of height suitable for the $\mathrm{UK}$ and Western Europe populations (the mean value is approximatly $1.70 \mathrm{~m}$ ). For populations with similar height distributions to those of the UK and Western Europe, this factor should be considered equal to 1 , otherwise the maximum acceleration can be obtained

Table 2 Coefficients for obtaining $\phi_{S l}$ in Eq. 12 for horizontal response $(y=1)$, where $x$ is the ratio between the pedestrian step and the span length, $i$ is a natural number greater than 1 , and $j=2 i-1$

\begin{tabular}{lll}
\hline $\boldsymbol{r}_{\boldsymbol{l}, \boldsymbol{n}}$ interval & $\boldsymbol{B}_{\mathbf{1}}$ & $\boldsymbol{B}_{\mathbf{2}}$ \\
\hline$[0.975,1]$ & $5460 x^{2}-820 x+14.34$ & 0.975 \\
{$[1,1.025]$} & & 1.025 \\
{$[j-0.025, j]$} & $1750 x^{2}-201 x+3.355$ & $j-0.025$ \\
{$[j, j+0.025]$} & & $j+0.025$ \\
\hline
\end{tabular}


Table 3 Coefficients of $\phi_{d}$ for Eq. 15 and vertical response

\begin{tabular}{lll}
\hline $\boldsymbol{r}_{\boldsymbol{v}, \boldsymbol{n}}^{*}$ interval & $\boldsymbol{C}_{\mathbf{1}}$ & $\boldsymbol{C}_{\mathbf{2}}$ \\
\hline$[0.95,1]$ & $\left(-50.9 \times 10^{4} \zeta^{3}+37.2 \times 10^{3} \zeta^{2}+\right.$ & 0.95 \\
{$[1,1.05]$} & $-1170 \zeta+5.05)$ & 1.05 \\
{$[1.95,2]$} & $\left(-254.5 \times 10^{3} \zeta^{3}+18.6 \times 10^{3} \zeta^{2}+\right.$ & 1.95 \\
{$[2,2.05]$} & $-585 \zeta+2.525)$ & 2.05 \\
{$[i-0.05,1]$} & $\left(-169.7 \times 10^{3} \zeta^{3}+12.4 \times 10^{3} \zeta^{2}+\right.$ & $i-0.05$ \\
{$[i, i+0.05]$} & $-390 \zeta+1.683)$ & $i+0.05$ \\
\hline
\end{tabular}

from the basic acceleration by using the factor $\phi_{p h}$, which is defined by Eq. 14, in which $h_{p}$ is the mean height of the target population in units of metres.

$$
\phi_{p h}=\frac{1.70}{h_{p}}
$$

\subsection{Factor related to the structural damping $\left(\phi_{d}\right)$}

Due to its different causes, damping is a difficult parameter to appraise. Nonetheless, several documents (Charles et al., 2006, Bachmann et al., 2006, and NA to BS EN 1991$2: 2003,2008$ ) have tried to quantify this value for traffic loading scenarios according to the material utilised in the structure. Generally suggested values for reinforced concrete structures are: $0.8-1.5 \%$, prestressed concrete: $0.5-1.0 \%$, composite sections (steel and concrete): $0.3-0.6 \%$, steel: $0.2-0.5 \%$ and timber structures: $1.0-1.5 \%$.

The basic accelerations have been obtained considering a damping ratio of $\zeta=0.5 \%$. For different damping ratios, the maximum acceleration can be obtained from the basic acceleration by using the factor $\phi_{d}$, which is defined by Eq. 15 and Tables 3 and 4 . Results show that the maximum accelerations registered are sensitive to the value of this parameter only near resonance (i.e., when the resonance parameters take values very close to a natural number) and for the first and second vibrational modes. Therefore, this factor $\phi_{d}$ needs to be assessed when the vertical or lateral resonance parameters linked to the first $\left(r_{v, 1}, r_{l, 1}\right)$ or the second mode $\left(r_{v, 2}, r_{l, 2}\right)$ are in the following intervals: $[i \pm 0.05]$, where $i$ is a natural number (i.e., $i=1,2,3,4, \ldots$ ). For the rest of the cases, $\phi_{d}=1$. The evaluation provided by this factor is valid for values of the damping ratio in the interval $0.002 \leq \zeta \leq 0.025$.

$$
\phi_{d}=\exp \left(C_{1} \times\left|r_{y, n}^{2}-C_{2}^{2}\right|\right), \quad y \in\{v, l\}
$$

\subsection{Factor related to the structural mass $\left(\phi_{s m}\right)$}

The parameter $\phi_{s m}$ is related to the number of spans and modal masses. The basic accelerations have been obtained for simply supported beams, where $\phi_{s m}=1$. For two and three span beams, this factor can be obtained from Fig. 5 .

Table 4 Coefficients of $\phi_{d}$ for Eq. 15 and horizontal response, where $j=2 i-1$ and $i$ is a natural number

\begin{tabular}{lll}
\hline$r_{l, n}^{*}$ interval & $C_{1}$ & $C_{2}$ \\
\hline$[0.95,1]$ & $\left(-147 \times 10^{4} \zeta^{3}+77.6 \times 10^{3} \zeta^{2}+\right.$ & 0.95 \\
{$[1,1.05]$} & $-1540 \zeta+6.29)$ & 1.05 \\
{$[j-0.05, j]$} & $\left(-49 \times 10^{4} \zeta^{3}+25.87 \times 10^{3} \zeta^{2}+\right.$ & $j-0.05$ \\
{$[j, j+0.05]$} & $-513.3 \zeta+2.097)$ & $j+0.05$ \\
\hline
\end{tabular}




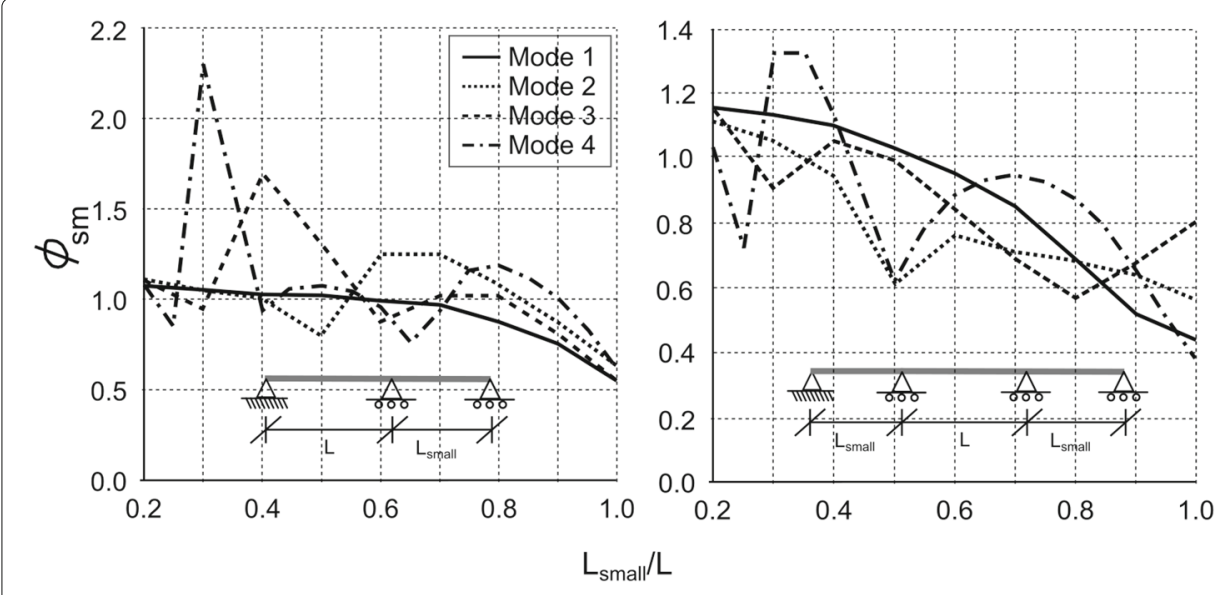

Fig. 5 Values for $\phi_{\text {sm }}$, according to mode and arrangement of spans

\section{Vertical and lateral accelerations caused by groups of pedestrians and continuous streams of pedestrians}

The issue of estimating the acceleration demands from groups or streams of pedestrians is significantly more complex than that of estimating the demands from a single pedestrian. When considering a single pedestrian, it is possible, and meaningful, to allocate the properties of that pedestrian so that they reflect the population being considered. However, when considering multiple pedestrians it has been shown that it is important to account for the significant inter-subject and intra-subject variability (Ramos et al., 2020). In Ramos et al. (2020), these issues are considered in a probabilistic manner, but the procedure is far more involved than what is desirable for preliminary design. For that reason, in what follows, a simple approach for obtaining first-order estimates of the demands from groups and streams is proposed.

\subsection{Group of pedestrians}

Under the assumption that all pedestrians within a group are identical, walk in a synchronised manner (same step frequency), and apply their load to the same point on the structure, then the vertical accelerations induced by this group will differ from those of a single pedestrian by a linear factor equal to the group size. That is, the maximum vertical acceleration would be defined as in Eq. 16.

$$
a_{v, g}=N a_{v}
$$

It should be noted that, when evaluating Eq. 16, the acceleration associated with the single pedestrian $a_{\nu}$ should be computed using a value of $\rho^{*}$ that accounts for the increased mass of pedestrians associated with the group (instead of the mass of a single pedestrian). That is, the effect of considering the group is not simply a linear scaling of the accelerations, but also accounts for a small shift in the resonance parameter. Some authors have observed that pedestrians modify the energy dissipation capacity of the structure (e.g., Brownjohn et al., 2004) in vertical or lateral direction, however the effect is not yet clear or quantified. Thus, this effect is disregarded in the implementation of this simple methodology. 
Despite the fact that the assumptions underpinning the above equation are often violated, we propose the use of this very simple expression during preliminary design.

Once one considers lateral accelerations, it is important to also consider the influence that pedestrian-structure interaction can have upon the responses from a group of pedestrians. In an event where pedestrians notice transverse movements of the deck, these may firstly adapt their gait to ensure that they do not loose the equilibrium. This widening of their step gait leads to an increase of the transverse loads introduced by pedestrians (further details of this nonlinear pedestrian-structure interaction can be found in Ramos et al., 2020). Larger lateral loads lead to a larger lateral footbridge response. For that reason, the expression we propose for evaluating the accelerations in the lateral direction is given by Eq. 17 in which an additional term $N_{n l}$, which accounts for these nonlinear interaction effects, is introduced. In Eq. 17, the lateral acceleration $a_{l}$ should be computed accounting for the mass of the entire group (that is, the group mass should influence $\rho^{*}$ and $\phi_{p m}$ ).

$$
a_{l, g}=\left(N+N_{n l}\right) a_{l}=\left(N+\frac{\beta}{\phi_{p m}}\right) a_{l}
$$

For a single pedestrian, the lateral force that they impart upon the bridge depends upon their own relative lateral acceleration with respect to the bridge as well as the global acceleration of the bridge (Ramos Moreno et al. 2020). For this single pedestrian case, these global accelerations are the sole result of this same pedestrian. However, when a group is considered, the global accelerations of the bridge result from the effects of each pedestrian and so the effects of this interaction do not scale linearly with the group size.

In order to account for this nonlinear interaction within a simple expression appropriate for preliminary design, an exercise was conducted in which the lateral accelerations were obtained using a 'scaled pedestrian' (the pedestrian mass is set to be $N$ times larger than the nominal single-pedestrian value, and the other assumptions regarding group behaviour used for the vertical case are retained). The accelerations obtained from this 'scaled pedestrian' are then compared with those found from an individual pedestrian in order to define an appropriate equivalent number of pedestrians $\left(N+N_{n l}\right)$ that account for the nonlinear interaction effects.

The term $N_{n l}$ is represented by the ratio $\beta / \phi_{p m}$ in order to enable the nonlinear interaction effect to be estimated for cases where the value adopted for $\phi_{p m}$ differs from that used to derive the values of $N_{n l}$. These nonlinear effects have only been found to be significant in the case that the resonance parameter $r_{l, 1}$ falls within two limited ranges of $[0.95,1.05]$ and $[2.9,3.1]$ or when the resulting peak lateral acceleration is larger than $0.15 \mathrm{~m} / \mathrm{s}^{2}$. In each of these two cases, the factor $\beta$ is found to be a function of the product of the group size and $\phi_{p m}$, as shown in Fig. 6.

In Fig. 6, $\beta=\beta_{1}$ is defined from Eq. 18, corresponding to the case that $0.95 \leq r_{l, 1} \leq 1.05$, and $\beta=\beta_{2}$ is relevant for $2.9 \leq r_{l, 1} \leq 3.1$ or when the peak acceleration is larger than $0.15 \mathrm{~m} / \mathrm{s}^{2}$ and is defined in Eq. 19. For any other value of the resonance parameter or when the total peak lateral acceleration is smaller or equal to $0.15 \mathrm{~m} / \mathrm{s}^{2}$, the response is assumed to be linearly proportional to the group size $(\beta=0)$.

$$
\begin{aligned}
& \beta_{1}=1.5 \times 10^{-4} x^{3}-4.5 \times 10^{-3} x^{2}+6 \times 10^{-2} x-0.15 \\
& \beta_{2}=5.0 \times 10^{-4} x^{2}-3.0 \times 10^{-3} x+7 \times 10^{-3}
\end{aligned}
$$




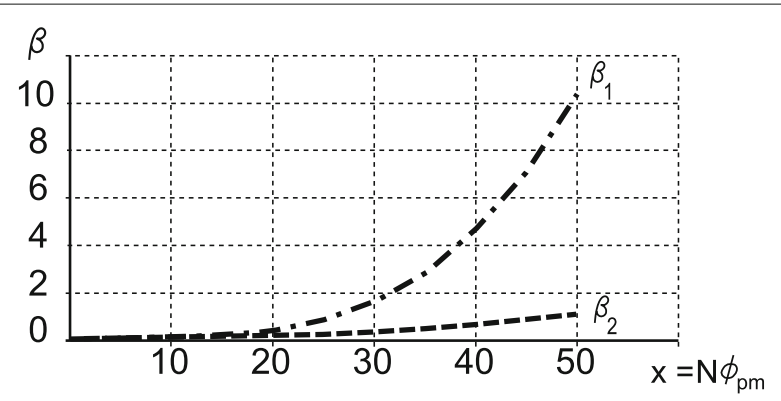

Fig. 6 Amplification factor $\beta$ for lateral response, $x=N \phi_{p m}$

\subsection{Continuous streams of pedestrians}

In order to enable designers to obtain estimates of the response of bridges under streams of pedestrians we couple the $\beta$ factors derived as part of this study with an existing approach advocated by NA to BS EN 1991-2:2003 (2008) and Setra (2006). The accuracy or validity of this approach was not assessed within the present study and a more elaborate approach to estimating the response due to streams of pedestrians is rather provided in Ramos Moreno et al. (2020).

The maximum vertical $\left(a_{v, s}\right)$ and lateral $\left(a_{l, s}\right)$ accelerations of a stream of pedestrians can be obtained as follows:

$$
\begin{aligned}
& a_{v, s}=N_{e q} \cdot \frac{a_{v}}{0.6} \\
& a_{l, s}=\left(N_{e q}+N_{n l}\right) \frac{a_{l}}{0.6}=\left(N_{e q}+\frac{\beta}{\phi_{p m}}\right) \frac{a_{l}}{0.6}
\end{aligned}
$$

where $N_{e q}$ is a number of "equivalent" pedestrians and can be adopted from Setra (2006) or NA to BS EN 1991-2:2003 (2008). The guideline SETRA (2006) defines this equivalence according to the density: for sparse or dense crowds (ped $\left./ \mathrm{m}^{2} \leq 0.8\right) N_{e q}=10.8 \sqrt{\zeta N}$ and for very dense crowds (ped $\left./ \mathrm{m}^{2}>0.8\right) N_{e q}=1.85 \sqrt{N}$, where $\zeta$ is the damping ratio and $N$ is the total number of pedestrians on the structure simultaneously. The factor $1 / 0.6$ is introduced to account for the fact that the flow is continuous as opposed to the event of a single pedestrian, and is taken from Grundmann et al. (1993).

The magnitude of the nonlinear factor $\beta$ for lateral loads depends on the equivalent number of pedestrians in a stream and is given again by Fig. 6, with $x=N_{e q} \phi_{p m}$.

It is worth highlighting that the load model considered for lateral loads is capable of reproducing the initial interaction that occurs when pedestrians sense a slight movement of the platform, but not an actual change of gait to adapt themselves better to the movement (named synchronisation, which would be a second phase of the interaction that apparently may take place between pedestrians and a platform).

\section{Verification of the methodology prediction of serviceability response}

In this section, the efficiency of the proposed methodology is assessed by comparing movements caused by pedestrians at real footbridges (experimentally recorded) with 
those predicted by the method. The similarity of the results highlights the competence of the method despite its simplicity.

\subsubsection{Footbridge over hringbraut, (Reykjavik, Iceland)}

The structure corresponds to a footbridge located in Reykjavik consisting of a postensioned concrete girder with 8 spans of longitudes between 15.4 and $27.1 \mathrm{~m}$. The transverse section is a slab with a width of $3.2 \mathrm{~m}$ (further details can be found in Gudmundsson et al., 2008).

Experimental measurements showed that the structure has a vertical frequency of $2.34 \mathrm{~Hz}$ and a damping ratio of value 0.006 . Tests of the concrete mix used demonstrated that the concrete Young's modulus had a value of $45 \mathrm{GPa}$ instead of $33.5 \mathrm{GPa}$ required in the project.

The proposed method is implemented considering only the three central longest spans with lengths of $15.5+27.1+15.5 \mathrm{~m}$ (in reality these have lengths of $20.6+27.1+23.6 \mathrm{~m}$ ). The length of the side spans results from an average value of the individual real lengths multiplied by $0.7\left(\frac{20.6+23.6}{2} \cdot 0.7\right)$. This factor 0.7 is applied to take into account that these have side spans that restrict their vibration (see Fig. 5). With these equivalent side spans, the three span footbridge assessed with the methodology is expected to have a dynamic behaviour similar to that of the real footbridge. This span disposition, the theoretical Young's modulus (considered in the project) and an additional mass for the balustrades, yields a vertical mode frequency of $2.06 \mathrm{~Hz}$ and if the real Young's modulus is considered the predicted vertical frequency is $2.38 \mathrm{~Hz}$.

A pedestrian of $800 \mathrm{~N}$ of weight walking on the bridge with vertical frequency $2.06 \mathrm{~Hz}$ at a step frequency of $2.06 \mathrm{~Hz}$ generates a peak vertical response of $0.37 \mathrm{~m} / \mathrm{s}^{2}$ and two pedestrians of the same weight $0.74 \mathrm{~m} / \mathrm{s}^{2}$. According to the measurements, at the real structure single pedestrians walking at a constant step frequency near resonance generated peak responses near $0.38 \mathrm{~m} / \mathrm{s}^{2}$ (except one case where peaks of $0.46 \mathrm{~m} / \mathrm{s}^{2}$ ) whereas two pedestrians caused peak accelerations of $0.71 \mathrm{~m} / \mathrm{s}^{2}$. The values measured in real tests and those predicted by this method are practically the same, with differences below $5 \%$ (Table 5).

\subsubsection{Aldeias footbridge, (Gouveia, Portugal)}

The footbridge consists of a steel box girder of variable depth and deck width of $1.5 \mathrm{~m}$ that spans a total distance of $57.8 \mathrm{~m}$ through three spans of longitude $17.7+30.0+10.1 \mathrm{~m}$ (further details can be found in Alves et al., 2008).

Designers of the bridge predicted numerically vertical modes at frequencies 3.13 and $4.50 \mathrm{~Hz}$, however dynamic tests at the structure once it was finished described vertical modes at frequencies 3.68 and $5.16 \mathrm{~Hz}$. For this footbridge, the proposed method is implemented considering that both side spans have the same length $(13.9 \mathrm{~m})$. This span

Table 5 Comparison of serviceability response experimentally recorded and serviceability response predicted by the methodology at a footbridge over Hringbraut, (Reykjavik, Iceland), where ped. corresponds to pedestrian

\begin{tabular}{lll}
\hline & Experimentally recorded $\mathrm{a}_{\boldsymbol{v}}$ & Proposed method $\mathrm{a}_{\boldsymbol{v}}$ \\
\hline 1 ped. $2.06 \mathrm{~Hz}$ & $0.38 \mathrm{~m} / \mathrm{s}^{2}$ & $0.37 \mathrm{~m} / \mathrm{s}^{2}$ \\
2 ped. $2.06 \mathrm{~Hz}$ & $0.71 \mathrm{~m} / \mathrm{s}^{2}$ & $0.74 \mathrm{~m} / \mathrm{s}^{2}$ \\
\hline
\end{tabular}


Table 6 Comparison of serviceability response experimentally recorded and serviceability response predicted by the methodology at Aldeias footbridge, (Gouveia, Portugal), where ped. corresponds to pedestrian

\begin{tabular}{lll}
\hline & Experimentally recorded $\mathbf{a}_{\boldsymbol{v}}$ & Proposed method $\mathbf{a}_{\boldsymbol{v}}$ \\
\hline 1 ped. $1.80 \mathrm{~Hz}$ & $<0.30 \mathrm{~m} / \mathrm{s}^{2}$ & $0.25 \mathrm{~m} / \mathrm{s}^{2}$ \\
\hline
\end{tabular}

disposition (together with the masses of the deck surfacing, the handrails and protection panels) describes a vertical mode at frequency $3.64 \mathrm{~Hz}$.

According to the proposed methodology, a pedestrian of $700 \mathrm{~N}$ of weight walking on the bridge at a step frequency of $1.80 \mathrm{~Hz}$ generates peak accelerations of $0.39 \mathrm{~m} / \mathrm{s}^{2}$ if the damping ratio considered is 0.006 (value commonly considered for steel box girders) or $0.25 \mathrm{~m} / \mathrm{s}^{2}$ if the magnitude of the damping ratio is 0.012 (introducing the effects of the large protection panels placed on this bridge that crosses over a highway). Real dynamic tests reflected that the damping ratio of the first vertical modes is moderately larger than 0.012 and that movements caused by a single pedestrian were below $0.30 \mathrm{~m} / \mathrm{s}^{2}$, see Table 6 (very similar to those predicted by the model with the second damping ratio).

\section{Verification of the serviceability design appraisal}

In this section, accelerations predicted using the detailed numerical analysis and those predicted by the simplified method just presented are compared. Generally speaking, an excellent agreement is found, as seen in what follows.

Figure 7 compares results obtained numerically and following the methodology to assess vertical responses of structures with two spans $(L+0.8 L)$ crossed by a pedestrian walking at $2.16 \mathrm{~Hz}$ and a step length of $0.65 \mathrm{~m}$ (the pedestrian weight is $700 \mathrm{~N}$ ) on structures with a T-slab section with a depth-to-span ratio $h / L=1 / 35$ and a damping ratio of $\zeta=0.005$ (i.e, for a set of parameters different to those considered in the reference case). The proximity of the simplified methodology results to those obtained numerically suggests that the accuracy of the methodology is good given its computational simplicity.

Figure 8 compares the maximum lateral accelerations obtained for simply supported structures calculated using the detailed numerical procedure and the simplified methodology presented in the paper. The structures considered have a composite box girder transverse section with $h / L=1 / 35$ and $\zeta=0.003$, whereas pedestrians were assumed to walk at a step frequency $f_{p}=2.16 \mathrm{~Hz}$ and with a step length of $0.65 \mathrm{~m}$. The results

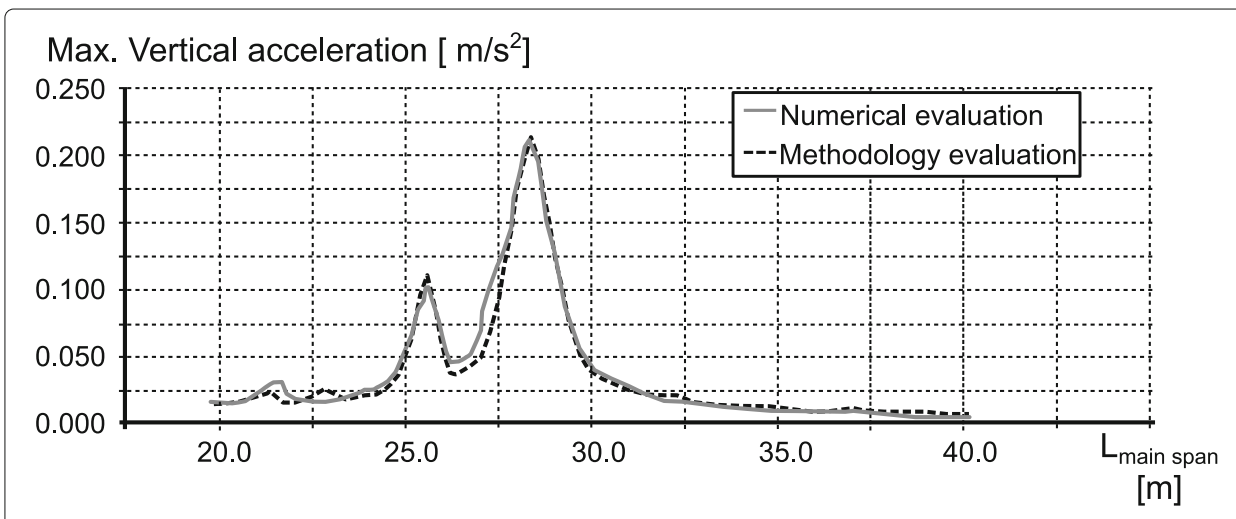

Fig. 7 Comparison of vertical response of two-span bridges, $L+0.8 L$ 


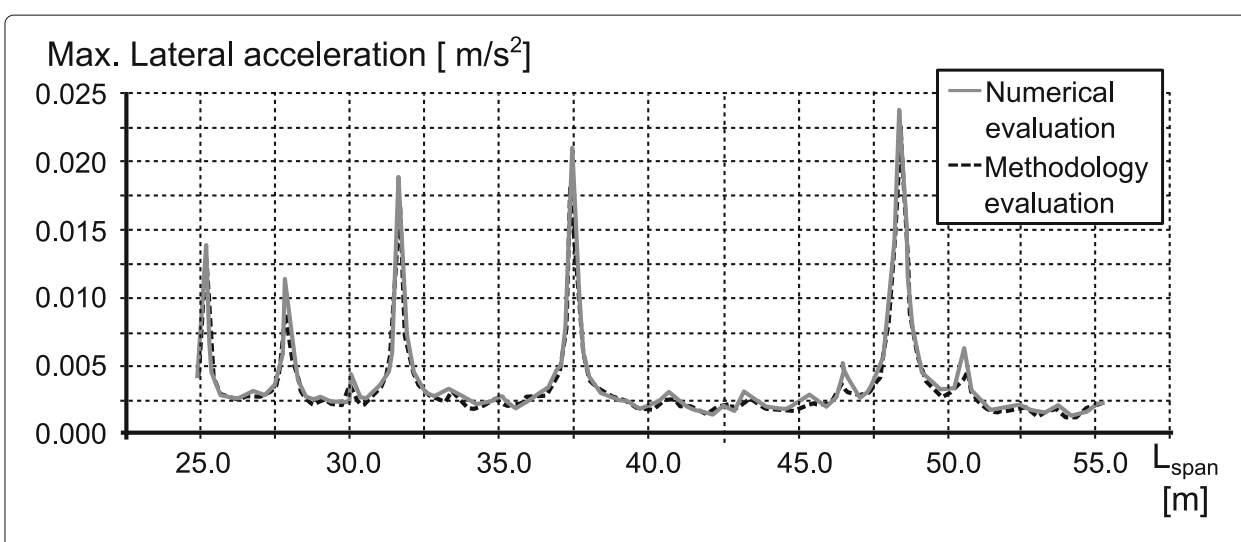

Fig. 8 Comparison of lateral response of simply supported bridges

shown in Fig. 8 again suggest that the simplified methodology proposed herein predicts the response with a very good degree of accuracy.

Figures 7 and 8 show that the accelerations registered in the deck could be very sensitive to the main span length and span arrangement (1, 2 or 3 spans). A sensitivity analysis should be included at the design stage in order to consider the uncertainty of these important parameters.

\section{Evaluation of the serviceability performance in conventional footbridges}

The new methodology presented herein constitutes a tool for evaluating the adequacy of the different design options usually considered in practice. The purpose of this section is to compare the different design options available for single span bridges with structural characteristics and materials listed in Fig. 3 and damping ratios of $\zeta=0.005$. The evaluation is performed considering the characteristics of a pedestrian $\left(m_{p}=71.36 \mathrm{~kg}\right)$ stream of commuters or at leisure with a density of $0.6 \mathrm{ped} / \mathrm{m}^{2}$ (the step frequency for the stream is the mean value provided in Fig. 2). For these analyses, it is considered that all sections have additional deck finishings and balustrades. Section S.6 also has as a non-structural concrete deck and sections S.7 to S.9 have non-structural wooden decks.

For a preliminary evaluation of the adequacy of the response in service, it can be considered that vertical accelerations in the range between 0.5 and $1.0 \mathrm{~m} / \mathrm{s}^{2}$ define the comfort limit, whereas, for lateral accelerations, responses in the range between 0.2 and $0.4 \mathrm{~m} / \mathrm{s}^{2}$ represent the limit of serviceable situations (further ranges can be found in codes and guidelines such as Setra, 2006 or NA to BS EN 1991-2:2003, 2008).

The results shown in Fig. 9 illustrate how the response of certain structures under the action of these pedestrian streams are large, and do not satisfy serviceability criteria for certain span lengths. However, it should be appreciated that although the values presented in Fig. 9 are often very large, in reality accelerations beyond $2 \mathrm{~m} / \mathrm{s}^{2}$, vertically, or $0.4 \mathrm{~m} / \mathrm{s}^{2}$, laterally, will probably not be developed due to a change of behaviour of pedestrians after sensing large vibrations (by either changing the characteristics of their walking, or even stopping).

It is also clear from Fig. 9 that there is a very strong dependence upon both the main span length just mentioned but also upon the pedestrian density. Given the strong sensitivity to these parameters, and the simplified nature of the approach proposed herein, 


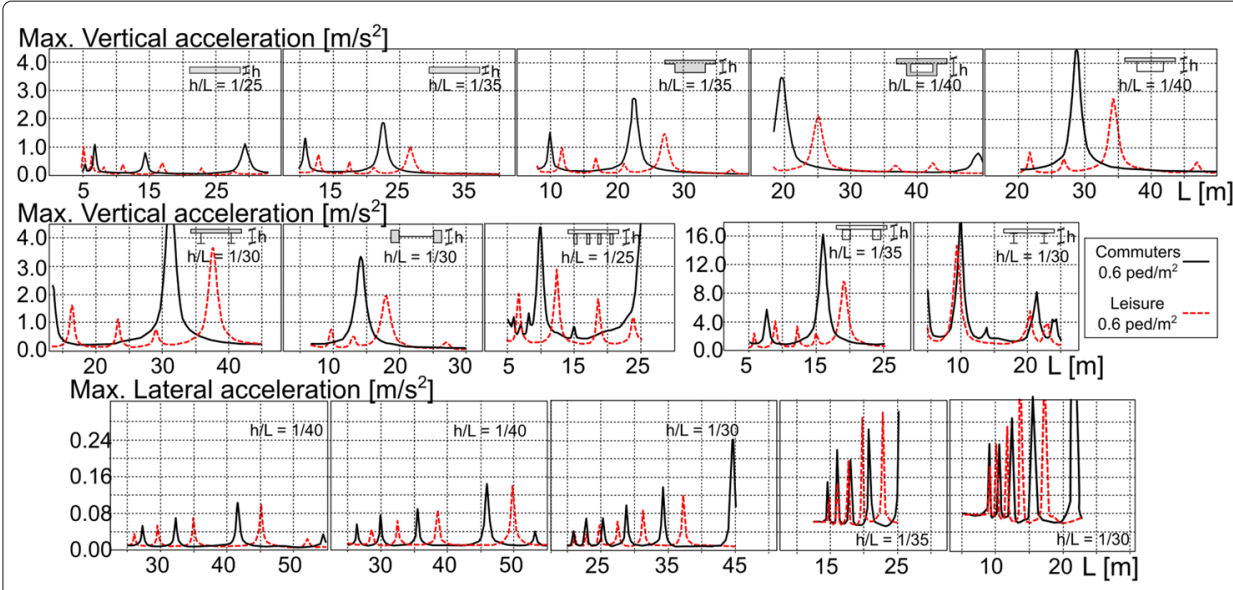

Fig. 9 Evaluation of serviceability of simply supported structures in vertical and lateral directions under the action of a pedestrian stream of density $0.6 \mathrm{ped} / \mathrm{m}^{2}$ with commuting or leisure being the aim of the journey

it is clear that any design decisions, even at preliminary stage, should account for uncertainties in these parameters. The uncertainty associated with the assumed damping ratio should also be considered.

Inspection of Fig. 9 also suggests that for this level of traffic, sections S.7 (aluminium) and S.9 (glass fiber reinforced polymers) have poor performance irrespective of span length. This suggests that for these types of decks it will be difficult to satisfy serviceability requirements using the slendernesses adopted here and for similar traffic.

For footbridges where this methodology shows that serviceability criteria may not be satisfied and responses may often be very large (in particular for sections S.7 and S.9), it is recommended that designers explore from an early design stage the benefits of implementing additional attenuation devices (e.g., tuned-mass dampers) as discussed in Garcia-Troncoso et al. (2020).

\section{Conclusions and future developments}

The paper presents a methodology for the serviceability evaluation of beam-type structures subjected to pedestrian loads. The steps of the procedure together with the fundamental underlying assumptions are outlined in this paper for both vertical and lateral response caused by these loads. Compared to current proposals, the method presented herein does propose an appraisal of the lateral accelerations generated by pedestrians in service, it is based on a realistic and experimentally contrasted description of the pedestrian loads, and it does not require the use of any software or elaborate analysis techniques.

Based on the methodological procedure, as well as the inherent assumptions and simplifications included for its development, it should be highlighted that:

a) An adequate evaluation of the response includes a comprehensive description of pedestrian loads and structural properties. Despite the fact that the method presented herein intends to simplify the assessment of pedestrian-induced vibrations, it still includes a refined evaluation of loads (both load magnitude and step frequencies). Regarding the structural properties, the method proposes evaluating the dynamic properties through a small number of parameters that are easy to appraise even during the early stages of design. 
b) The prediction of the lateral response attempts to include the interaction phenomenon that has been detected for pedestrian loading scenarios through a parameter that reflects a certain response nonlinearity. This parameter shows that under certain circumstances the nonlinear response in the lateral direction can be larger than that obtained considering only linear results. However, it should be highlighted that this model is only able to reproduce what seems to be a first stage of what might happen in reality (it is not reflecting a change of step frequency according to the response generated, or synchronisation). This phenomenon could be further investigated for its inclusion in the assessment of lateral structural response. Although having said that, it is the opinion of the authors that serviceability is unlikely to be satisfied when it becomes necessary to model these types of phenomena.

c) The comparison of the response described by the proposed methodology to that recorded in real footbridges highlights the excellent competence of the method in describing the serviceability response of footbridges at very early stages of their design.

d) The comparison of the response for a set of structures calculated both using an advanced numerical procedure and the proposed simplified approach shows that the methodology is an adequate tool for the evaluation of vertical and lateral structural response for preliminary design.

e) The estimates of the accelerations are extremely sensitive to the span length and the type of traffic loading scenario. For conventional sections commonly considered within design, once values of the span length and pedestrian loading are defined, the method clearly identifies span ranges that should be avoided.

f) Finally, it is important to recognise that the simplicity of the proposed approach allows a designer to obtain estimates of accelerations with very little computational effort. The proposed method therefore lends itself to undertaking analyses in which the sensitivity of the results to various design assumptions is quantified. The strong sensitivity of the results shown in this article imply that such sensitivity analyses are an indispensable component of the design process.

\section{Supplementary Information}

The online version contains supplementary material available at https://doi.org/10.1186/s43251-020-00012-9.

Additional file 1: Additional file can be found in a repository in Open Science Framework Dataset repository. The additional file consists of an Excel table that lists the values of $a_{, n}^{b}$ and $a_{l, n}^{b}$ according to the values of $f_{p, v}$ and $r_{v, n}$ (or $f_{p, l}$ and $r_{l, n}$ ) as described in the paper.

Additional file 2: Additional file located in a repository in Open Science Framework Dataset repository is also included hereunder as a table listing the values of $a_{, n}^{b}$ and $a_{l, n}^{b}$ according to the values of $f_{p, v}$ and $r_{v, n}\left(\right.$ or $f_{p, l}$ and $r_{l, n}$ ) as described in the paper.

Abbreviations

FE: finite elements

\section{Acknowledgements}

The authors are grateful for the support provided by La Caixa to fund the PhD studies of the first author at Imperial College London. 


\section{Funding}

The work developed in this paper is partially supported by La Caixa.

\section{Availability of data and materials}

The datasets used to derive the current study are available from the corresponding author on reasonable request. The datasets used to implement the methodology presented in the current study are included in this published article.

\section{Competing interests}

The authors declare that they have no competing interests.

\section{Author details}

${ }^{1}$ Arcadis Consulting UK, London, UK. ${ }^{2}$ Department of Civil and Environmental Engineering, Imperial College London, South Kensington Campus, SW7 2AZ London, United Kingdom.

Received: 17 September 2020 Accepted: 26 October 2020

Published online: 29 December 2020

\section{References}

AASHTO (2009) LRFD guide specifications for the design of pedestrian bridges. Standard, American Association of State Highway and Transportation Officials (AASHTO), Washington DC, USA

Alves R, Barbosa F, Caetano E (2008) The Aldeias footbridge at Gouveia: design, construction and dynamic behaviour. In: Caetano E, Cunha A (eds). Proceedings of the Third International Conference, Footbridge 2008

Bachmann H, Amman W, Deischl F, et al. (1995) Vibration problems in structures: practical guidelines. Birkhauser Verlag, Basel

Bertram JEA, Ruina A (2001) Multiple walking speed-frequency relations are predicted by constrained optimization. J Theor Biol 209:445-453

Brownjohn J, Fok P, Roche M, Omenzetter P (2004) Long span steel pedestrian bridge at Singapore Changi Airport - Part 2: crowd loading tests and vibration mitigation measures. Struct Eng 82:7

BS 5400 (1978) Steel, concrete and composite bridges. Specification for loads. Appendix C. Standard, British Standards Institution (BSI), London, UK

Buckingham E (1914) On physically similar systems; illustrations of the use of dimensional equations. Phys Rev 4(4):345-376

Butz C, Feldmann M, Heinemeyer C, et al. (2008) Advanced load models for synchronous pedestrian excitation and optimised design guidelines for steel footbridges. Technical report, Research Fund for Coal and Steel

CAN/CSA-S6-06 (2006) Canadian highway bridge design code. Standard, Canadian Standards Association (CSA), Mississauga, ON, Canada

Charles P, Hoorpah W, Boniface V, et al. (2006) Footbridges. Assessment of vibrational behaviour of footbridges under pedestrian loading. Practical guidelines. Technical report, SETRA/AFGC

Dallard P, Fitzpatrick A, Flint A, Le Bourva S, Low A, Ridsdill Smith R, M Willford M (2001) The London Millennium footbridge. Struct Eng 79(22):17-33

Dassault Systèmes (2013) Abaqus CAE FEA

EN 1991-2:2003 (2003) Eurocode 1: Actions on structures - Part 2: Traffic loads on bridges. Standard, European Committee For Standardization (CEN), Brussels, Belgium

Firth I, Cooper D (2002) New materials for new bridges - Halgavor bridge, UK. Struct Eng Int 12(2):80-83

Garcia-Troncoso N, Ruiz-Teran A, Stafford P (2020) Attenuation of pedestrian-induced vibrations in girder footbridges using tuned-mass dampers. In: Advances in Bridge Engineering (accepted)

Grundmann H, Kreuzinger H, Schneider M (1993) Schwingungsuntersuchungen für Fußgängerbrücken [In German]. Bauingenieur 68:215-225

Gudmundsson G, Ingólfsson E, Einarsson B, Bessason B (2008) Serviceability assessment of three lively footbridges in Reykjavik. In: Caetano E, Cunha A (eds). Proceedings of the Third International Conference, Footbridge 2008, Porto, Portugal, 2008

Macdonald J (2009) Lateral excitation of bridges by balancing pedestrians. Proc R Soc A-Math Phys Eng Sci 465:1055-1073

NA to BS EN 1991-2:2003 (2008) UK National Annex to Eurocode 1: Actions on structures. Part 2: Traffic loads on bridges. Standard, British Standards Institution (BSI), London, UK

Pachi A, Ji T (2005) Frequency and velocity of people walking. Struct Eng 83(3):36-40

Pedersen L, Frier C (2010) Sensitivity of footbridge vibrations to stochastic walking parameters. J Sound Vib 329:2683-2701

Ramos Moreno C, Ruiz-Teran A, Stafford P (2020) Impact of Stochastic Representations of Pedestrian Actions on Serviceability Response. In: Proceedings of the Institution of Civil Engineers - Bridge Engineering. https://doi.org/10 1680/jbren.19.00050

Weidmann U (1993) Transporttechnik der Fussgänger - Transporttechnische Eigenschaften des Fussgängerverkhers (Literaturauswertung). [In German]. Technical report, Institut für Verkehrsplanung, Transporttechnick, Strassen und Eisenbahnbau, ETH Zurich

\section{Publisher's Note}

Springer Nature remains neutral with regard to jurisdictional claims in published maps and institutional affiliations. 